\title{
ESTUDO DO EQUILÍBRIO LÍQUIDO-VAPOR DO SISTEMA ETANOL -ÁGUA - NITRATO DE CÁLCIO TETRA- HIDRATADO A PRESSÃO NORMAL
}

\author{
C. Leal ${ }^{1}$, W. A. P. Magalhães ${ }^{1}$, R. B. Soares ${ }^{1}$, M. F. Mendes ${ }^{1}$ \\ ${ }^{1}$ Universidade Federal Rural do Rio de Janeiro, Departamento de Engenharia Química \\ Laboratório de Termodinâmica Aplicada e Biocombustíveis \\ E-mail para contato: marisamf@ufrrj.br
}

\begin{abstract}
RESUMO - Este trabalho visa a obtenção de dados experimentais de equilíbrio líquido-vapor do sistema ternário etanol-água-nitrato de cálcio, à pressão atmosférica. Vários solventes já foram estudados para a quebra do azeótropo do sistema águaetanol, incluindo sais ( $\mathrm{NaCl}, \mathrm{CaCl}_{2}$, acetatos de sódio de potássio), solventes orgânicos (benzeno, etilenoglicol, etc.) e líquidos iônicos. A escolha deste sal deve-se à falta de informações na literatura sobre o comportamento desse sistema e a importância da busca de outros solventes mais baratos para a separação etanol-água. Com isso, os dados foram medidos usando-se um ebuliômetro do tipo Othmer e as análises de composição foram feitas usando-se um densímetro digital. Os experimentos foram realizados usando-se uma quantidade mínima fixa do sal de 0,06 molar, variando-se a concentração de etanol na solução, em triplicata. Os resultados foram modelados usando-se UNIQUAC e apresentaram um desvio relativo médio inferior a 7\%, tanto na composição da fase vapor, quanto na temperatura. Esse estudo mostrou que o nitrato de cálcio é um solvente que pode ser utilizado para a produção de etanol anidro com uma pureza elevada, por promover a quebra do azeótropo formado na mistura etanolágua.
\end{abstract}

\section{INTRODUÇÃO}

A maior demanda por energia promove um aumento do preço do petróleo bruto, afetando diretamente a atividade econômica global. Todos estes motivos levaram ao desenvolvimento de pesquisas por fontes de energia alternativa, renovável, sustentável, eficiente, de baixo custo e com menores emissões de contaminantes e gases de efeito estufa. Entre as muitas alternativas encontram-se os biocombustíveis, sendo uma fonte de energia menos agressiva ao meio ambiente, além de ser uma escolha vantajosa devido à sua capacidade de renovação, biodegradabilidade e geração de gases de qualidade aceitável (Nigam \& Singh, 2011).

O etanol anidro, atualmente, tem evoluído muito no mercado industrial, principalmente na área de combustíveis, como auxiliar na gasolina, fazendo com que o aumento do número de octanagem ocorra e promova uma queima superior do combustível, reduzindo assim a emissão de 


\section{9 a 22 de outubro de 2014 \\ Florianópolis/SC}

$\mathrm{CO}$ e de particulados. Em se tratando de um composto isento de nitrogênio, os compostos nitrogenados da combustão são reduzidos. Porém, o etanol hidratado, que antecede o processo da desidratação, provoca formação de duas fases quando misturado à gasolina, resultando em problemas no motor (Gomis et al., 2006). Isso faz com que seja necessário o processo de desidratação, para isentar o etanol da água, tornando-o anidro e ideal para sua utilização.

O principal processo de obtenção de etanol é a partir da cana-de-açúcar, que posterior a retirada de impurezas, ocorre a fermentação alcoólica, apresentando como produto a mistura de etanol e água. Desta forma, para a obtenção do álcool em sua forma anidra é necessário que esta mistura etanol/água passe por um processo de desidratação. O que impede a facilidade de separação desta mistura é a formação de azeótropo de mínimo neste sistema, onde ocorre na composição 89,4 mol\% etanol a 351,35 K à pressão atmosférica (Kumar et al., 2010). Esse azeótropo impede a separação completa do etanol por destilação simples, tendo assim, uma concentração máxima possível de se obter por destilação simples à pressão atmosférica.

Uma das etapas que mais consome energia dentro do processo de produção de etanol é a destilação. Em função disso, pesquisas sobre novas tecnologias para a desidratação do etanol vêm sendo desenvolvidas (Huang et al., 2008).

Em geral, de acordo com Soares (2010), adiciona-se um solvente e a técnica é denominada destilação extrativa ou azeotrópica, dependendo da volatilidade da substância. A destilação extrativa consiste na adição de um solvente de baixa volatilidade em altas concentrações, acima da alimentação e que permanece em contato com o líquido na coluna. $\mathrm{O}$ objetivo é aumentar mais a volatilidade de um dos componentes do que a do outro, fazendo possível a separação. Já na destilação azeotrópica, o solvente adicionado é mais volátil que os componentes da mistura, sendo retirado no topo da coluna. A tecnologia mais utilizada para separação etanol-água é a destilação extrativa e sua eficiência depende, em grande parte, do solvente extrativo empregado (Huang et al., 2008).

A destilação extrativa com sais dissolvidos ocorre em solução líquida por dois componentes líquidos miscíveis e afeta a atividade de algum dos componentes da mistura. De acordo com Llano-Restrepo \& Aguilar-Arias (2003), o sal se associa preferencialmente com moléculas de um dos componentes, permitindo a separação por destilação. Ainda, segundo Pinto (2000), essa técnica tem sido estudada até a atualidade e emprega menor relação de energia/massa de etanol anidro.

O presente trabalho tem como objetivo fazer experimentalmente a curva de equilíbrio do etanol-água com nitrato de cálcio tetra-hidratado para verificar sua eficiência no uso da destilação extrativa, e assim, a eficiência deste processo para a desidratação do etanol. 


\section{MATERIAIS E MÉTODOS}

\subsection{Materiais}

Nitrato de cálcio tetra-hidratado (Vetec Química Fina Ltda, Rio de Janeiro, Brasil), etanol absoluto (99.8\% de pureza mínima, Vetec Química Fina Ltda, Rio de Janeiro, Brasil) e água destilada foram usados para preparar as misturas.

Souza et al. (2013) mediram algumas propriedades termofísicas do etanol e da água previamente, garantindo sua pureza.

\subsection{Metodologia Experimental}

Os dados experimentais foram medidos através de misturas de água-etanol em frações molares de 0.2 a 0.95 de etanol. Já o nitrato de cálcio tetra-hidratado foi utilizado na concentração de 0.06 mol. $\mathrm{L}^{-1}$. O valor de concentração do nitrato foi escolhido visando a comparação com outros sais, que já foram testados na mesma concentração.

Os experimentos foram realizados utilizando um ebuliômetrodo tipo Othmer, em vidro, como apresentado na Figura 1. Este ebuliômetro é composto por uma célula de equilíbrio, dois condensadores e duas saídas para retirar amostras das fases líquidas e vapor. O volume total da célula de equilíbrio é $300 \mathrm{~mL}$, onde a mistura ocupava aproximadamente $50 \mathrm{~mL}$. No condensador de serpentina há uma abertura para o meio externo, garantindo a pressão atmosférica (1 atm). As temperaturas das fases líquidas e vapor foram medidasatravés de termômetros digitais com $\pm 0,1$ ${ }^{\circ} \mathrm{C}$ de incerteza. Os experimentos foram realizados em triplicata.

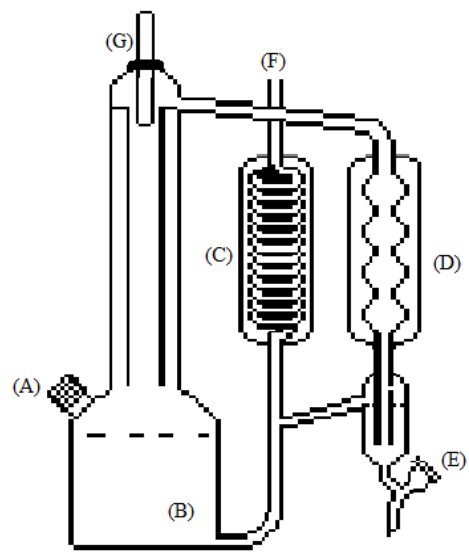

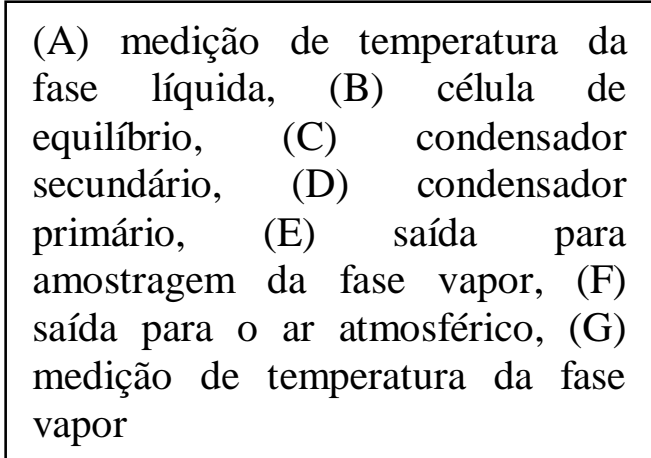

Figura 1 - Diagrama esquemático do ebuliômetro.

O procedimento experimental começa com a pesagem das massas, estipuladas de acordo com a fração molar dos componentes da mistura água-etanol e da concentração do sal predefinida. Após a pesagem, a carga é introduzida na célula de equilíbrio juntamente com um agitador magnético, adicionado para garantir a total dissolução do sal no meio. Após o início da 
circulação de água pelos condensadores, o sistema, isolado, é posto sob agitação durante 5 minutos e após este, a manta de aquecimento é ligada. A temperatura da fase vapor é medida em intervalos de 10 minutos até que se obtenham quatro valores iguais consecutivos, onde se considera que o estado de equilíbrio foi atingido. Neste momento, ainda com o fluxo de água e o aquecimento ocorrendo, coleta-se uma amostra da fase vapor para análise no densímetro digital. Cada ponto foi analisado em triplicata.

\subsection{Análises das amostras}

As composições das amostras foram determinadas com as medidas experimentais de massa específica, obtidos utilizando um densímetro digital (GEHAKA, modelo DSL 920), com \pm $0,0001 \mathrm{~g} / \mathrm{cm}^{3}$ de incerteza. Uma curva de calibração foi previamente construída com a mistura água-etanol em composições molares conhecidas, em triplicata. A curva de calibração obtida foi utilizada para determinar a fração molar de etanol nas amostras (Souza et al., 2013).

\subsection{Modelagem matemática}

A equação UNIQUAC (Universal quase-Chemical), de Reid et al. (1987), descrita em Smith et al. (2000), leva em conta dois termos, um combinatorial (para levar em conta o tamanho e as diferenças de forma das moléculas) e um residual (para levar em conta as interações moleculares), sendo escrita pela equação 1:

$$
\ln \gamma_{i}=\ln \gamma_{i}+\ln \gamma_{i}^{R}
$$

sendo $\gamma$ o coeficiente de atividade, os sobrescritos $C$ e $R$ os indicativos de combinatorial e residual, respectivamente, e o subscrito $i$ o indicativo da espécie presente no sistema.

Diversos métodos para a predição do efeito salino sobre o equilíbrio líquido-vapor de misturas de solventes podem ser usados. Grande parte dos modelos está fundamentada em equações termodinâmicas utilizadas em sistemas não-eletrolíticos, fazendo-se uso de um ou mais termos adicionais (empíricos ou teóricos) referentes à contribuição do eletrólito. Seader et al. (1986) apresentaram um modelo, para o cálculo do efeito salino sobre o equilíbrio líquido-vapor, que combina a equação UNIQUAC modificada com um termo Debye-Hückel, que reflete a atração inter-iônica. A consideração fundamental deste modelo é que todos os eletrólitos estão completamente dissociados. Neste modelo, de acordo com a equação 2, o coeficiente de atividade $\gamma_{n}$ de um solvente $n$ é:

$$
\ln \gamma_{n}=\ln \gamma_{n}^{D-H}+\ln \gamma_{n}^{C}+\ln \gamma_{n}^{R}
$$

sendo o primeiro termo, $\ln \gamma_{n}{ }^{D-H}$, o termo Debye-Hückel e os demais são iguais ao modelo UNIQUAC. Os parâmetros de interação entre um íon $i$ e um solvente $m\left(a_{i m}\right.$ e $\left.a_{m i}\right)$, propostos pelos autores, formam as equações 3: 


$$
a_{i m}=a^{*}{ }_{i m}+\theta_{i \cdot} \sum_{j=i} \delta_{i j, m} \cdot \theta_{j} \text { e } a_{m i}=a^{*}{ }_{m i}+\theta_{i} \cdot \sum_{j=i} \delta_{i j, m} \cdot \theta_{j}
$$

Nestas equações, $a^{*}{ }_{i m}$ e $a^{*}{ }_{m i}$ representam os parâmetros de interação de referência e $\delta_{i j, m}$ é um parâmetro de interação entre os íons e solvente. O somatório é de todas as espécies, exceto $i$, e $\theta_{i}$ corresponde à fração de área superficial do íon $i$. Macedo et al. (1990) realizaram um estudo para o cálculo do equilíbrio de fases para eletrólitos. $\mathrm{O}$ modelo proposto se baseia no trabalho de Seader et al. (1986). O termo UNIQUAC não foi modificado e o termo Debye-Hückel é o mesmo proposto pelos autores em 1923. O termo Debye Hückel, para a energia de Gibbs em excesso, é dado pela equação 4:

$$
G_{D-H}^{* E}=\frac{-k}{3 \cdot \varepsilon} \cdot \sum_{i=1}^{N S O L U} s_{i} \cdot z_{i}^{2} \cdot e^{2} \cdot \tau \cdot(k a)
$$

sendo $s_{i}$ o número de íons do tipo $i$ no sistema, $z_{i}$ o número da carga do íon $i, e$ a carga do elétron, $\varepsilon$ a constante dielétrica da mistura de solventes, $a$ a distância Debye Hückel de maior proximidade dos íons, $N S O L U$ o número de espécies iônicas, $\eta$ a função do comprimento Debye reduzido (ka), e $k$ o inverso do comprimento Debye, fornecido pela equação 5:

$$
k^{2}=\frac{8 \cdot \pi \cdot N_{0} \cdot d_{S} \cdot I \cdot e^{2}}{(\varepsilon \cdot K \cdot T)}
$$

sendo, além do que já foi definido, $N_{o}$ o número de Avogrado, $I$ a força iônica, $T$ a temperatura, $K$ a constante de Boltzmann e $d_{s}$ a densidade da mistura dos solventes. Pela derivação da equação 4, obtém-se o termo Debye Hückel para o coeficiente de atividade de um solvente, e a equação 6 em uma mistura de solventes:

$$
\ln \gamma_{n}^{D-H}=\frac{V_{n}}{24 \cdot \pi \cdot N_{0} \cdot a^{3}} \cdot(k a)^{3} \cdot \sigma \cdot(k a)
$$

Tendo $V_{n}$ o volume parcial molar do solvente $n$ e $\sigma$ uma função do comprimento reduzido Debye Hückel, escrito como a equação 7:

$$
\sigma(k a)=\frac{3}{(k a)^{3}} \cdot\left[1+k a-\frac{1}{1+k a}-2 \cdot \ln (1+k a)\right]
$$

Fixando $a=4 \times 10^{-10} \mathrm{~m}$ e combinando as equações de 5-7, obtém-se a equação 8 em que o termo correto para a contribuição Debye Hückel, para o coeficiente de atividade de um solvente $n$ :

$$
\ln \gamma_{n}^{D-H}=\frac{2 \cdot A \cdot M_{n} \cdot d_{s}}{b^{3} \cdot d_{n}} \cdot\left[1+b \cdot \sqrt{I}-\frac{1}{1+b \cdot \sqrt{I}}-2 \cdot \ln (1+b \cdot \sqrt{I})\right]
$$

Sendo pelas equações 9 e 10 : 


$$
\begin{aligned}
& A=\frac{1,327757 \cdot 10^{5} \cdot d_{s}^{\frac{1}{2}}}{(\varepsilon . T)^{\frac{3}{2}}} \\
& b=\frac{6,359696 \cdot d_{s}^{\frac{1}{2}}}{(\varepsilon \cdot T)^{\frac{1}{2}}}
\end{aligned}
$$

As densidades $d s$ e $d n$ são aplicadas em $\mathrm{kg} / \mathrm{m}^{3}$ e a temperatura em K. A densidade molar da mistura de solventes $d$ 's $\left(\mathrm{kmol} / \mathrm{m}^{3}\right)$ é calculada de acordo com a seguinte equação empírica 11:

$$
d_{i}^{\prime}=\frac{1}{\left(\sum_{n} \frac{x_{n}}{d_{n}^{\prime}}\right)}
$$

Sendo $x_{n}^{\prime}$ a fração molar (em base livre de sal) do solvente $n$ na fase líquida. A constante dielétrica, da mistura dos solventes, é obtida a partir dos valores para os solventes puros, utilizando a regra de mistura de Oster, descrita em Macedo et al. (1990) pela equação 12:

$$
\varepsilon=\varepsilon_{1}+\left[\frac{\left(\varepsilon_{2}-1\right) \cdot\left(2 \cdot \varepsilon_{2}+1\right)}{2 \cdot \varepsilon_{2}-\left(\epsilon_{1}-1\right)}\right] \cdot x_{2}^{\prime} \cdot \frac{V_{2}}{V}
$$

\section{RESULTADOS E DISCUSSÕES}

A Tabela 1 apresenta os dados de ELV do sistema etanol-água-nitrato de cálcio, em base livre de sal, a pressão normal, preditos pelo modelo apresentado e a Tabela 2 apresenta os dados medidos nesse trabalho, com os desvios padrão das medidas e o desvio relativo entre as medidas experimentais e preditas. Observa-se que os desvios relativos foram muito baixos, o que evidencia o bom comportamento do modelo frente aos dados experimentais.

Para poder comprovar a eficiência do sal na quebra do azeótropo, a Figura 2 apresenta os dados do sistema água-etanol (Dechema) e os dados medidos nesse trabalho.

Tabela 1 - Dados de ELV para o sistema de estudo, com 0,06 mol.L ${ }^{-1}$ de concentração de sal

\begin{tabular}{cccccc}
\hline $\mathrm{x}$ & $\mathrm{y}$ & $\mathrm{T}\left({ }^{\circ} \mathrm{C}\right)$ & $\mathrm{x}$ & $\mathrm{y}$ & $\mathrm{T}\left({ }^{\circ} \mathrm{C}\right)$ \\
\hline 0,99 & 0,997 & 77,6 & 0,37 & 0,774 & 77,5 \\
0,89 & 0,965 & 77,3 & 0,27 & 0,718 & 78,5 \\
0,78 & 0,929 & 77,1 & 0,17 & 0,667 & 80,1 \\
0,68 & 0,884 & 77,1 & 0,06 & 0,513 & 86,9 \\
0,57 & 0,851 & 77,1 & 0,01 & 0,155 & 98,4 \\
0,47 & 0,803 & 77,4 & & & \\
\hline
\end{tabular}


Tabela 2 - Dados de ELV para o sistema etanol-água-nitrato de cálcio tetra-hidratado

\begin{tabular}{ccccccc}
\hline $\mathrm{X}$ & $\mathrm{y}$ & $\mathrm{T}\left({ }^{\circ} \mathrm{C}\right)$ & $\mathrm{DP}{ }^{*} \mathrm{y}$ & $\mathrm{DP} * \mathrm{~T}$ & $\mathrm{DR} * * \mathrm{y}(\%)$ & $\mathrm{DR} * * \mathrm{~T}(\%)$ \\
\hline 0,20 & 0,6813 & 81,8 & 0,0287 & 0,0070 & 2,14 & 2,12 \\
0,40 & 0,7713 & 80,8 & 0,0200 & 0,0030 & 0,35 & 4,26 \\
0,60 & 0,7936 & 79,4 & 0,0090 & 0,0021 & 1,17 & 2,58 \\
0,80 & 0,9132 & 79,0 & 0,0162 & 0,0006 & 1,70 & 2,46 \\
0,90 & 0,9476 & 78,1 & 0,0090 & 0,0006 & 1,80 & 1,30 \\
0,95 & 0,9732 & 75,9 & 0,0106 & 0,0006 & 1,76 & 3,10 \\
\hline
\end{tabular}

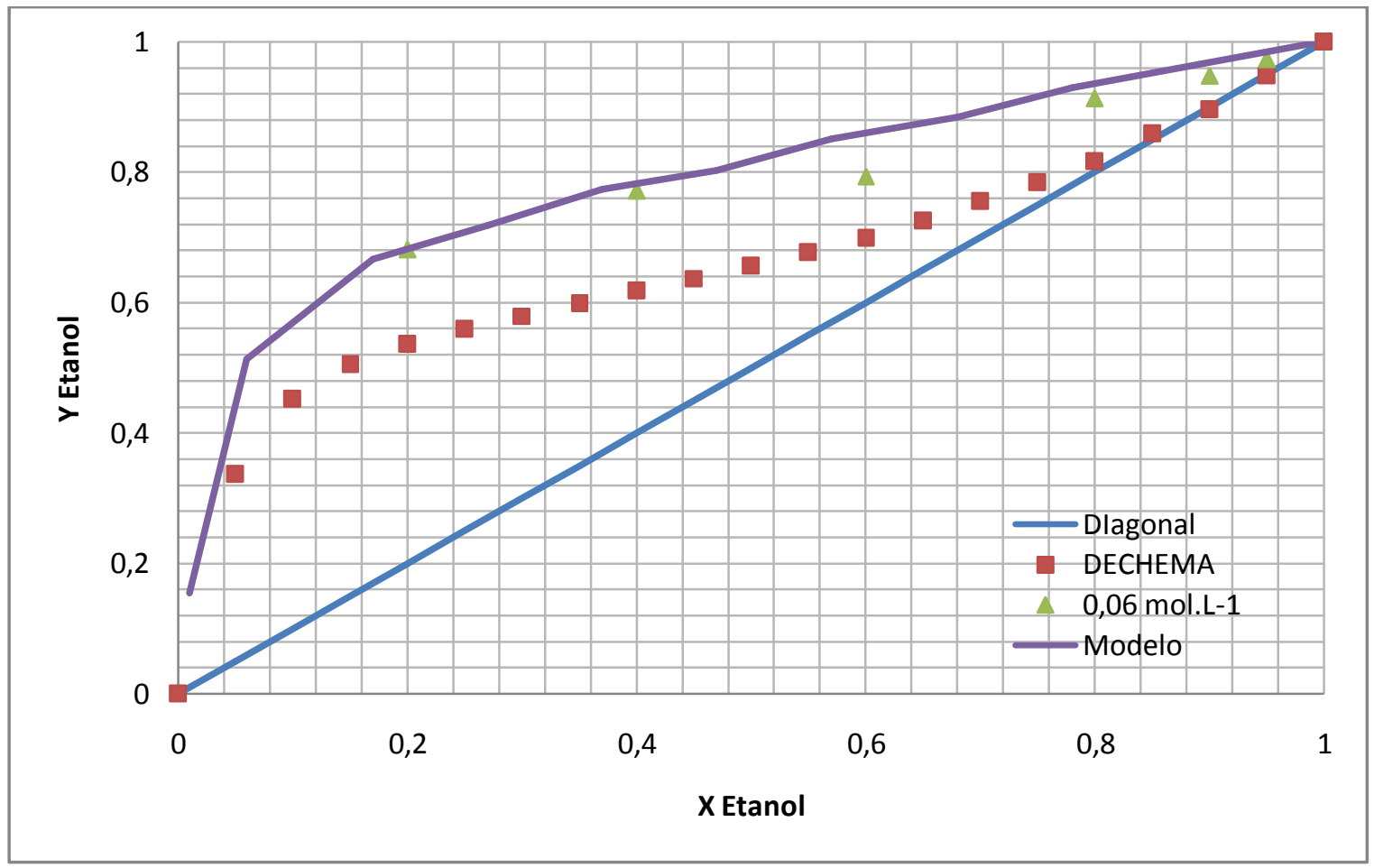

Figura 2 - Diagrama T-x-y para o sistema etanol-água-nitrato de cálcio tetra-hidratado a 0,06 mol.L ${ }^{-1}$ comparado ao sistema água-etanol, modelo e Dechema (1981).

\section{CONCLUSÃO}

Neste trabalho, dados inéditos de ELV foram gerados para o sistema ternário água-etanolnitrato de cálcio, a pressão atmosférica. Observou-se que o sistema se comportou de forma satisfatória e houve um enriquecimento da fase vapor em etanol, ocorrendo a quebra do azeótropo. Os dados experimentais foram satisfatórios, tendo em vista que o desvio relativo da temperatura $(2,63 \%)$ e da composição de etanol na fase vapor $(1,49 \%)$ foram baixos, comparativamente com o modelo aplicado. 


\section{REFERÊNCIAS}

DECHEMA. VAPOR-LIQUID EQUILIBRIUM DATA COLLECTION, Aqueous-Organic Systems (Supplement 1), v. 1, part 1a. GMEHLING, V. J.; ONKEN, U.; ARLT, W. Frankfurt/M, 1981.

GOMIS V., FRONT A., SAQUETE M.D. Vapour - liquid - liquid and vapour - liquid equilibrium of the system water + ethanol + heptane at $101.3 \mathrm{kPa}$. Fluid Phase Equil., 248, p. $206-210,2006$.

HUANG, H.; RAMASWAMY, S.; TSCHIRNER, U. W.; RAMARAO, B. V. A review of separation technologies in current and future biorefineries. Separation and Purification Technology, v.62, p. 1-21, 2008.

KUMAR, S.; SINGH, N.; PRASAD, R. Anhydrous ethanol: a renewable source of energy. Renewable and Sustainable Energy Reviews, v. 14, p. 1830-1844, 2010.

LLANO-RESTREPO, M.; AGUILAR-ARIAS, J. Modeling and simulation of saline extractive distillation columns for the production of absolute ethanol. Computer \& Chemical Engineering, v. 27, p. 527-549, 2003.

MACEDO, E. A.; SKOVBORG, P.; RASMUSSEN, P. Calculation of phase equilibria for solutions of strong electrolytes in solvent-water mixtures. Chem.Engin. Science, v. 45(4), p. 875-882, 1990.

NIGAM, P.; SINGH A. Production of liquid biofuels from renewable resources. Progress in Energy and Combustion Science, v. 37, p. 52-68, 2011.

PINTO, R.; WOLF MACIEL, M.; LINTOMEN, L. Saline extractive distillation process for ethanol purification. Computers and Chemical Engineering, v. 24, p. 1689-1694, 2000.

REID, R. C.; PRAUSNITZ, J. M., POLING, B. E. The properties of gases \& liquids. 4ed, McGraw-Hill, New York, 1987.

SEADER, B.; FREDENSLUNG, A.; RASMUSSEN, P. Calculation of vapor-liquid equilibria in mixed solvent/salt systems using an extended UNIQUAC equation. Chem. Engin. Science, v. 41(5), p. 1171-1183, 1986.

SOARES, R. B. Estudo da destilação extrativa salina em coluna recheada para a produção de etanol. Tese de Mestrado. 2010. 98f. Dissertação em Engenharia Química - Instituto de Tecnologia, Universidade Federal Rural do Rio de Janeiro, Seropédica.

SMITH, J. M.; VAN NESS, H. C.; ABBOTT, M. M. Introdução à termodinâmica da engenharia química, 5 ed.Rio de Janeiro, Editora LTC, 2000.

SOUZA, W.L.R., SILVA, C.S., MELEIRO, L.A.C., MENDES, M.F. Vapor-liquid equilibrium of the (water + ethanol + glycerol) system: Experimental and modelling data at normal pressure J. Chem. Thermod. 67, p. 106-111, 2013. 